\title{
Precipitation signatures of ground-based VLF transmitters
}

\author{
P. Kulkarni, ${ }^{1}$ U. S. Inan, ${ }^{1}$ T. F. Bell, ${ }^{1}$ and J. Bortnik ${ }^{2}$ \\ Received 30 May 2007; revised 14 December 2007; accepted 18 January 2008; published 23 July 2008.
}

[1] Numerical raytracing with Landau damping is used to calculate $>100 \mathrm{keV}$ electron precipitation signatures induced by hypothetical VLF transmitters distributed broadly in geomagnetic latitude and operating at a wide range of frequencies. A one-half second pulse from each source is simulated and attenuation to the base of the magnetosphere for geomagnetic latitudes from $10^{\circ}$ to $60^{\circ}$ is calculated. Source location affects induced precipitation more strongly than operating frequency or radiated power. Sources located at $35^{\circ}$ to $45^{\circ}$ induce the most $>100 \mathrm{keV}$ precipitation for the 10 to $40 \mathrm{kHz}$ waves typical of ground-based VLF sources, while locations below $\lambda \simeq 15^{\circ}$ or above $\lambda \simeq 55^{\circ}$ are least effective at precipitating energetic electrons. In all cases, induced precipitation increases as the operating frequency decreases, with $10 \mathrm{kHz}$ waves from a source at $\lambda \simeq 35^{\circ}$ the most effective at precipitating $>100 \mathrm{keV}$ electrons. Precipitation signatures produced by five existing ground-based VLF transmitters are also simulated: the NAA, NLK, NAU, NPM, and NWC VLF transmitters. NWC induces the strongest $>100 \mathrm{keV}$ electron precipitation signature, followed by NPM, NAU, NAA, and NLK.

Citation: Kulkarni, P., U. S. Inan, T. F. Bell, and J. Bortnik (2008), Precipitation signatures of ground-based VLF transmitters, J. Geophys. Res., 113, A07214, doi:10.1029/2007JA012569.

\section{Introduction}

[2] Resonance interactions between very low frequency (VLF) Whistler mode waves and energetic electrons are believed to play a significant role in the loss of trapped particles in the near-Earth space environment [Kennel and Petschek, 1966; Lyons et al., 1972; Abel and Thorne, 1998a]. Such waves occur naturally, in the form of e.g., plasmaspheric hiss, VLF chorus and lightning-generated whistlers, and are also injected into the radiation belts by powerful ground-based VLF transmitters. These transmitters operate continuously radiating signals that illuminate the Earth-ionosphere waveguide for naval communication, but the wave energy inevitably leaks into near-Earth space where the injected waves interact with and precipitate radiation belt electrons. Previous authors [Inan et al., 1984; Abel and Thorne, 1998a, 1998b] have attempted to quantify the energetic electron precipitation induced by ground-based VLF sources.

[3] Inan et al. [1984] used a test-particle simulation to determine the spatial distribution of electron precipitation caused by existing ground-based VLF transmitters. While exclusively considering only propagation along the magnetic field lines (i.e., within ducts of enhanced ionization) this study concluded that transmitter geographic location and operating frequency strongly affects precipitation zone size. Abel and Thorne [1998a, 1998b] calculated electron lifetimes driven by wave-particle interactions using bounce-averaged

\footnotetext{
${ }^{1}$ STAR Laboratory, Stanford University, Stanford, California, USA.

${ }^{2}$ Department of Atmospheric and Oceanic Sciences, University of California, Los Angeles, California, USA.

Copyright 2008 by the American Geophysical Union. 0148-0227/08/2007JA012569\$09.00
}

pitch angle diffusion coefficients and concluded that VLF transmitters have a substantial effect on energetic $(>100 \mathrm{keV})$ electron lifetimes at $L<2.6$.

[4] In this paper we build upon the results presented by Inan et al. [1984] by removing the restriction of ducted propagation, and instead use the Stanford 2D VLF Raytracing program [Inan and Bell, 1977] to determine raypaths in a smooth magnetosphere. We use the method of [Bortnik et al., 2006b] to determine energetic electron precipitation signatures induced by five existing groundbased VLF sources as well as five different hypothetical transmitters distributed broadly in geomagnetic latitude with a wide range of operating frequencies. For the existing VLF transmitters, we use geomagnetic coordinates in the centered dipole model that correspond to the source the geographic latitudes. These results allow us to determine the wave frequencies and transmitter locations that most effectively precipitate $>100 \mathrm{keV}$ electrons. We interpret the numerically determined global signatures of transmitter induced particle precipitation in terms of the physics of VLF wave propagation (both in the Earth-ionosphere waveguide and in the magnetosphere) and the location and effectiveness of wave-particle interaction regions.

\section{Model Description and Simulation Procedure}

[5] Table 1 shows the properties of the five existing VLF transmitters considered in this study. To calculate the energetic $(>100 \mathrm{keV})$ electron precipitation induced by VLF transmitters, we utilize four separate models: (1) antenna radiation pattern and attenuation of VLF wave energy versus distance from source for an electrically small vertical ground-based VLF antenna, (2) attenuation (mainly due to collisional absorption in the $D$-region) of VLF wave energy 
Table 1. Parameters of the Five Existing VLF Transmitters Considered in This Study

\begin{tabular}{lccc}
\hline \multicolumn{1}{c}{ List of VLF Transmitters } & \\
\hline Transmitter & Geomagnetic Latitude, deg & Radiated Power, $\mathrm{kW}$ & Frequency, $\mathrm{kHz}$ \\
\hline NAA (Cutler, Maine) & $54.6^{\circ} \mathrm{N}$ & 1000 & 24.0 \\
NLK (Jim Creek, Washington) & $52.9^{\circ} \mathrm{N}$ & 192 & 24.8 \\
NAU (Aguadilla, Puerto Rico) & $28.6^{\circ} \mathrm{N}$ & 100 & 40.75 \\
NPM (Lualuaiei, Hawaii) & $21.4^{\circ} \mathrm{N}$ & 424 & 21.4 \\
NWC (N. W. Cape, Australia) & $31.7^{\circ} \mathrm{S}$ & 1000 & 19.8 \\
\hline
\end{tabular}

during propagation through the ionosphere to the base of the magnetosphere, (3) ray tracing determination of the configuration of raypaths within the magnetosphere, using magnetic field and cold plasma density models, and accounting for Landau damping along raypaths as determined by the suprathermal particle distribution, and (4) pitch angle scattering and precipitation of energetic electrons in resonance interactions with nonducted VLF Whistler mode waves, with the precipitation flux levels dependent upon the efficiency of scattering but also on the assumed trapped electron distribution function near the loss cone. We examine the first three relatively simple models together, and separately discuss the modeling of the resonant interaction, pitch angle scattering and resulting electron precipitation.

\subsection{Antenna Radiation, Attenuation, and Ray Propagation}

[6] For each source the resultant precipitation is calculated by launching rays representing a one-half second long pulse at the base of the magnetosphere, taken to be $1000 \mathrm{~km}$, at points spaced $0.25^{\circ}$ ranging from $10^{\circ}$ to $60^{\circ}$ in geomagnetic latitude. This latitude range captures all of the induced $>100 \mathrm{keV}$ precipitation that is of interest, because injections at very low $\left(\sim 10^{\circ}\right)$ or very high $\left(\sim 60^{\circ}\right)$ latitudes induce relatively little precipitation (see below). To quantitatively model the precipitation induced by the injected waves, we must determine the input power at $1000 \mathrm{~km}$ altitude, based on VLF-antenna radiation properties and taking into account ionospheric absorption losses.

[7] Rodriguez et al. [1994] conducted an extensive study of the ionospheric modification caused by ground-based VLF transmitters. Because of the long $(10-15 \mathrm{~km})$ wavelengths of waves radiated by such sources, the antennas can be analyzed as short, vertical monopoles on a ground plane. However, reflections from the conducting plane allows us to treat a monopole of height $h / 2$ as a dipole of height $h$ [Rodriguez, 1994, p. 63, and references therein]. The formula for the radiated Poynting flux for a dipole of height, $h$, is given as:

$$
S(r, \theta)=\frac{3 P_{\mathrm{tot}} \sin ^{2} \theta}{4 \pi r^{2}}
$$

where $P_{\text {tot }}$ represents total transmitter radiated power, $\theta$ is the angle measured from the vertical dipole axis, and $r$ the radial distance from the dipole axis to the measurement point [Rodriguez, 1994, p. 65, equation 4.6]. We use the above formula to calculate the Poynting flux at the base of the ionosphere, taken to be at $100 \mathrm{~km}$, at points ranging from $10^{\circ}$ to $60^{\circ}$ latitude with $r$ measured from the source location.
[8] From the base of the ionosphere, the wave energy can be assumed to propagate vertically through the ionosphere to the base of the magnetosphere (from 100 to $1000 \mathrm{~km}$ ), and is attenuated according to a nighttime absorption factor taken from Helliwell [1965, Figures 3-35]. This collisional absorption in fact occurs over the altitude range of $\sim 75-120 \mathrm{~km}$, where the imaginary part of the refractive index (representing attenuation due to collisional losses) is large [Helliwell, 1965, p. 65, Figures 3-29], and is dependent on the particular ionospheric profile in effect at the time. However, for our purposes here we simply need to account for the absorption losses in a generic manner to capture its magnitude in an average sense but more importantly its dependence on geomagnetic latitude. We thus use an interpolation of the results given by Helliwell [1965, Figures 3-35], including the dependence on geomagnetic latitude. In this way, our results incorporate the different absorption that would occur for different transmitter locations and operating frequencies. More specifically, our location-dependent precipitation signatures include the effects of higher absorption at $20^{\circ}$ versus $50^{\circ}$, and $20 \mathrm{kHz}$ versus $10 \mathrm{kHz}$. However, we do not include the $3 \mathrm{~dB}$ polarization loss, as this is constant for all locations and transmitters and thus does not affect our comparative analysis of the effects of different sources. For the purpose of this study, we assume a smoothly varying ionosphere without horizontal density gradients so that the wave normal angles at $1000 \mathrm{~km}$ are largely vertical. After entering the magnetosphere, the rays propagate in a manner determined by the cold plasma density and Earth's magnetic field gradients.

[9] The Stanford VLF raytracing program [Inan and Bell, 1977] uses a centered dipole model for the magnetic field with an electron gyrofrequency of $880 \mathrm{kHz}$ at the ground at the equator. Above $L=2.25$, we use the Carpenter and Anderson [1992] model under geomagnetically quiet conditions for the cold plasma density, with a plasmapause at $L=5.5$, with $d=0, t=2, K_{p(\max )}=0$, and $\bar{R}=90$ for day of year, time of day, maximum $K_{p}$ in the preceding $24 \mathrm{~h}$ and average 13-month sunspot number. Below $L=2.25$, we model the cold plasma electron density with the diffusive equilibrium model of Angerami and Thomas [1964]. While traversing the magnetosphere, rays also undergo pathintegrated Landau damping, which we calculate based on the velocity space distribution function specified by Bell et al. [2002]. In our raytracing, we neglect upgoing rays formed by the shadow boundary in the conjugate hemisphere, as well as refractive effects that would focus and defocus the rays thereby modifying the power flux.

[10] To summarize thus far, VLF wave energy radiates from a ground-based source in accordance with the formula given by (1). We calculate Poynting flux values for rays at $100 \mathrm{~km}$ altitude, every $0.25^{\circ}$ from $10^{\circ}$ to $60^{\circ}$ in geomag- 


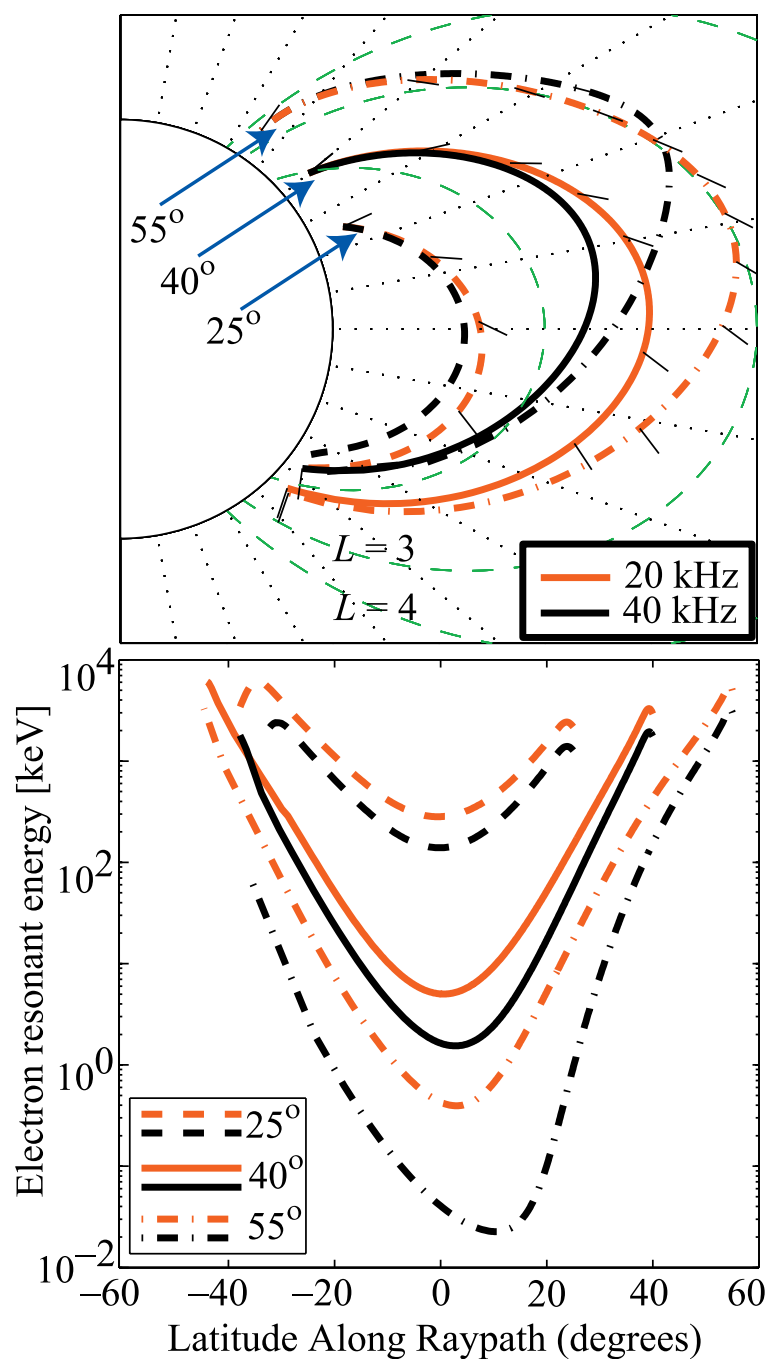

Figure 1. (a) $20 \mathrm{kHz}$ and $40 \mathrm{kHz}$ rays injected from geomagnetic latitudes of $25^{\circ}, 40^{\circ}$, and $55^{\circ}$. In each case, the red ray is $20 \mathrm{kHz}$, and the black ray is $40 \mathrm{kHz}$. Every 50 time steps, we have indicated the direction of the wave normal angle on 3 of the rays with black lines. (b) Resonant energy versus geomagnetic latitude along raypath for the rays above. The legend is in the lower left. Note that rays injected from higher latitudes resonate with lower energy electrons.

netic latitude. The attenuation of the wave energy due to collisional absorption during trans-ionospheric propagation (up to $1000-\mathrm{km}$ ) is separately accounted for, and the rays, which are traced for a one-half second pulse duration, are injected into the magnetosphere with vertical wave normal angles at $1000 \mathrm{~km}$. We utilize numerical raytracing to determine raypaths through the magnetosphere and include Landau damping of the wave energy as a function of distance along the raypath. The wave energy propagating in the magnetosphere interacts with the energetic electrons in cyclotron resonance, leading to the pitch angle scattering and precipitation of the energetic electrons as described below.

\subsection{Wave-Particle Interaction and Electron Precipitation}

[11] The basic gyroaveraged equations of motion for resonance interactions between obliquely propagating whistler mode waves and energetic electrons are given by Bell [1984]. We use the method of Bortnik et al. [2006a], to integrate these equations to calculate the pitch angle changes induced by VLF transmitter signals considered here. While the methodology described by Bortnik et al. [2006a] was applied to a transient lightning stroke, we have made appropriate modifications to allow us to model a onehalf second long pulse.

[12] Specifically, we combine extensive raytracing, Landau damping and interpolation to produce a wave map that contains the relevant parameters (refractive index, wave power, wave normal angle) at $1^{\circ}$ intervals along each field line from -40 to $40^{\circ}$ in geomagnetic latitude. At each $1^{\circ}$ interval range, these wave characteristics are used to compute the root mean square (i.e., averaged over Larmor phase) pitch angle change for loss cone electrons with parallel velocities in a narrow range around the resonant parallel velocity at that location. We assume that the interactions are linear (i.e., wave intensity is not large enough to phase-trap the electrons) and that the interactions between adjacent latitude bins are independent. To determine the total flux of precipitated particles, we apply the calculated pitch angle changes to near loss cone electrons (i.e., to electrons immediately above the loss cone), recognizing that their scattering is similar to that which was calculated for the electrons at the loss cone. We use a square (i.e., sharp) pitch angle distribution and model the initial (unperturbed) trapped energetic electron distribution function as by Bortnik et al. [2006a]:

$$
f(v)=\frac{a_{1}}{v_{m}^{4}}-\frac{a_{2}}{v_{m}^{5}}+\frac{a_{3}}{v_{m}^{6}}
$$

where $f$ is in units of $\mathrm{s}^{3} \mathrm{~cm}^{-6}, v_{m}$ is $v / \sqrt{1-v^{2} / c^{2}}$, and $a_{1}$, $a_{2}$ and $a_{3}$ are constants respectively equal to $4.9 \times 10^{5} \mathrm{~cm}^{-2}$ $\mathrm{s}^{-1}, 8.3 \times 10^{14} \mathrm{~cm}^{-1} \mathrm{~s}^{-2}$, and $5.4 \times 10^{23} \mathrm{~s}^{-3}$.

[13] The total cumulative pitch angle scattering, as described by Bell [1984], depends largely on the duration of time that the electron remains in phase with wavefields. The electron pitch angle also changes adiabatically along its trajectory along a given field line, and energetic electrons are in principle trapped indefinitely on this field line in the absence of wave forces [Walt, 1994, p. 42]. The total pitch angle change is thus a combination of the waveinduced pitch angle change, which occurs during the wave-particle interaction, and the adiabatic change. Only wave forces, however, can bring about a change in the equatorial pitch angle (i.e., the electron pitch angle referred to the equator) which otherwise remains constant in the course of the adiabatic motion. The wave-particle resonance condition is given by:

$$
v_{z}=\frac{m \omega_{\mathrm{H}} / \gamma-\omega}{k_{z}}
$$

where $v_{z}$ is the resonant electron velocity along the Earth's magnetic field, $\mathbf{B}_{0}, m$, an integer, is the resonant mode, $\omega_{\mathrm{H}}$ is the electron gyrofrequency, $\gamma=1 / \sqrt{\left(1-v^{2} / c^{2}\right)}, \omega$ is the wave frequency, and $k_{z}$ the component of the wave $k$-vector along $\mathbf{B}_{0}$ [Bell, 1984]. Because $\gamma$ is a function of total electron velocity, $v$, equation (2) is not an explicit formula. We can therefore write $v_{z}=v \cos \alpha$ and solve the resulting quadratic equation to calculate resonant electron velocity for 
near loss cone electrons. Figure 1 shows the outcome of this calculation, with the explicit formula used being equation (2) by Bortnik et al. [2006b].

[14] Equation (2) illustrates the manner in which location of the interaction (which determines $\omega_{\mathrm{H}}$ ) and $\omega$ affect resonant velocity: $v_{z}$ is generally inversely proportional to $\omega$ and directly proportional to $\omega_{\mathrm{H}}$. For electron energies of $1 \mathrm{MeV}$, however, relativistic effects imply that there is no such simple dependence, and our code properly accounts for the relativistic gamma factor. In Figure 1, we examine $20 \mathrm{kHz}$ and $40 \mathrm{kHz}$ rays injected at geomagnetic latitudes of $25^{\circ}, 40^{\circ}$, and $55^{\circ}$, and calculate $v_{z}$ along the raypath, where the short black lines on each raypath represent the direction of the $k$-vector at selected discrete points. For a given source location, the $40 \mathrm{kHz}$ ray resonates with lower energy electrons and crosses the geomagnetic equatorial plane at lower $L$-shells. Rays injected by any ground-based VLF source tend to cross the magnetic equatorial plane at an $L$-shell such that the wave frequency is less than or equal to half the electron gyrofrequency [Inan and Bell, 1991]. Because gyrofrequency is larger closer to the Earth's surface, a $40 \mathrm{kHz}$ ray crosses the equator at a lower $L$-shell compared to a $20 \mathrm{kHz}$ ray. Figure 1 also shows that higher source latitudes lead to lower resonant energy along the raypath because the ray propagates to higher $L$-shells where $\omega_{H}$ is lower. For example, a $40 \mathrm{kHz}$ ray injected from $25^{\circ}$ resonates with more energetic electrons than a $20 \mathrm{kHz}$ ray injected from both $40^{\circ}$ and $55^{\circ}$.

[15] This last result implies that source location may be more important than operating frequency in determining induced energetic electron precipitation. However, although location does influence resonant velocity more than wave frequency, the resultant electron precipitation also depends on factors such as wave power flux and wave normal angle. These factors are in turn affected by gradients in ionospheric density, latitude-dependent trans-ionospheric absorption and Landau damping.

[16] The methodology just described, i.e., calculation of pitch angle change of near loss cone electrons in resonance with the wave, allows us to determine the precipitation signatures that would be observed at $100 \mathrm{~km}$ altitude over a range of $L$-shells in the source hemisphere. For the existing VLF transmitters considered, we scale these results in longitude (by simply using $r^{-2}$ dependence of wave power density) to determine precipitation zones on the Earth's surface by using the methodology of Bortnik et al. [2006b], where the precipitated flux as a function of longitude simply scales with distance from the source location [Ibid, Figure 6].

[17] We focus herein on the first-order counterstreaming resonance between the wave and energetic electrons to more clearly capture and elucidate the essential physics and the dependencies on different parameters. Higher-order resonance modes are generally less efficient resonant interactions with higher energy electrons which are fewer in number. Simulation results demonstrate that including other resonant modes, $m,-5 \leq m \leq 5$ does not significantly (less than an order of magnitude) affect the calculated precipitation signatures at $L \lesssim 2.6$. Below we compare the induced precipitation signature with including all resonance modes versus the fundamental $m=1$ mode for a source located at $\lambda=35^{\circ}$. Including all resonance modes results in precipitation in both the northern and southern hemispheres, which are properly calculated in our analysis. While the flux values are larger at $L>2.6$, below $L=2.6$ there is very little difference (see below). This result indicates that the contributions due to higher order resonances do not significantly affect precipitation at $L<2.6$ which is the region of maximum precipitation for 10 to $40 \mathrm{kHz}$ signals and $>100 \mathrm{keV}$ electrons of interest here. Below we also explore in greater detail the sensitivity of our results to the different model assumptions.

[18] We should also note that in our simulations we specifically calculate precipitation induced by a one-half second long VLF pulse. The actual transmitters, however, operate continuously, and thus may have long-term effect on the pitch angle distribution of energetic electrons that are not revealed in our calculations. Nevertheless, a recent study by Shprits et al. [2006] documented that electron lifetimes are primarily governed by the value of pitch angle scattering close to the loss cone. Accordingly, it is likely that the induced precipitation of loss cone energetic electrons as is calculated here may indeed yield useful information regarding the long-term effects of VLF transmitters. However, this study only examines the effect of a short pulse on a very small range of angles near the loss cone, which will not directly affect the entire distribution. If we were considering continuous precipitation then we would have to examine the complete pitch angle distribution and work toward a diffusive equilibrium solution. This issue should be examined in greater detail in future studies.

[19] We stress that our calculated precipitation signatures result from considering wave frequencies of $10 \mathrm{kHz}$ to $40 \mathrm{kHz}$ and only $>100 \mathrm{keV}$ electrons. Wave frequencies of $3-5 \mathrm{kHz}$, e.g., undergo numerous magnetospheric reflections and would yield precipitation at substantially different $L$-shells. On the other hand, $10-40 \mathrm{kHz}$ waves make only a single traverse of the magnetosphere and do not propagate beyond $L \simeq 3$. Furthermore, focusing on electrons with energies $>100 \mathrm{keV}$ makes $10 \mathrm{kHz}$ waves appear more effective because, as shown in Figure 1, $40 \mathrm{kHz}$ waves often resonate with $<100 \mathrm{keV}$ electrons.

[20] We now compare and contrast the energetic electron precipitation induced by hypothetical ground-based VLF sources distributed in geomagnetic latitude and operating frequency. We wish to highlight the underlying physics that leads to our numerical results, and to understand and elucidate the effects of varying transmitter location, operating frequency and radiated power. The remaining results for the actual VLF transmitters can then be explained in a straightforward manner in terms of the established dependencies.

\section{Simulation Results and Analysis}

[21] We begin by displaying raypaths calculated by the Stanford 2D VLF raytracing program [Inan and Bell, 1977] for 20 and $40 \mathrm{kHz}$ waves, with all rays injected with vertical wave normal angles at the base of the magnetosphere. The raypaths shown in Figure 2 define the overall envelope of precipitation that would be observed at $100 \mathrm{~km}$. Note that, as in Figure 1, $40 \mathrm{kHz}$ illuminate a smaller region of the magnetosphere and cross the equatorial plane at lower $L$-shells. Although the raypaths shown are not different for different source locations and radiated power, the power flux and wave normal angle deposition do vary with these 

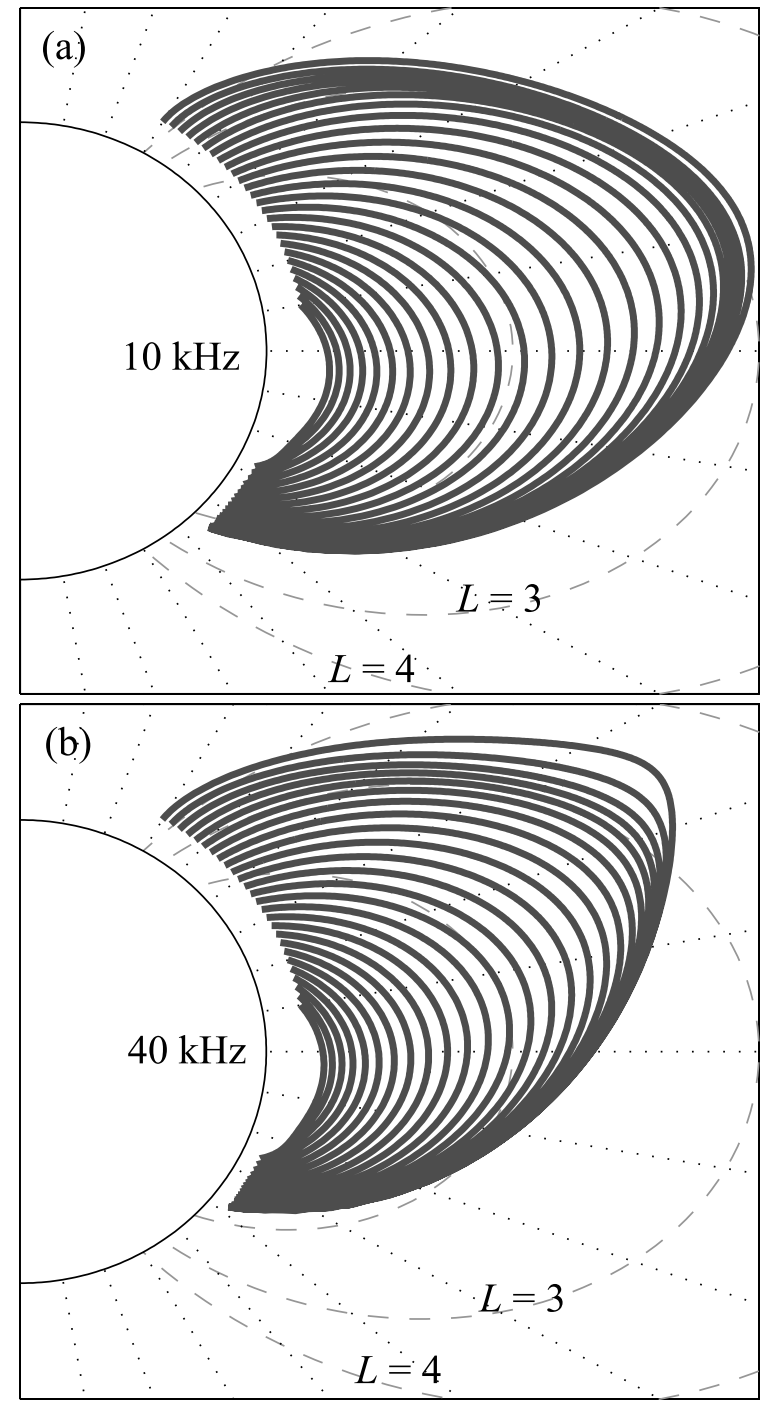

Figure 2. Raypaths for $20 \mathrm{kHz}$ and $40 \mathrm{kHz}$ sources, where rays were injected from $10^{\circ}-60^{\circ}$ with initial vertical wave normal angles at the base of the magnetosphere. The plasmapause was located at $L=5.5$ in this figure, and for most of our simulation results. Note that the $40 \mathrm{kHz}$ rays bend in toward the Earth more sharply.

parameters. Figure 3 shows the wave power flux and wave normal angle distribution injected into the magnetosphere by $500 \mathrm{~kW}, 20 \mathrm{kHz}$ sources located at $25^{\circ}$ and $55^{\circ}$. While the resolution may not be high enough to discern the differences, more power is in fact injected closer to the source site, due to the latitude-dependent Helliwell absorption [Helliwell, 1965, Figures 3-35]. This absorption factor contributes to the relatively weak wave power deposited at low latitudes, as shown in the top row. Also note that, in both cases, the wave normal angle becomes highly oblique $\left(\sim 80^{\circ}\right)$ in the southern hemisphere. The resultant $>100 \mathrm{keV}$ precipitation signatures depend on these parameters as well as electron resonant energy and pitch angle scattering efficiency, all of which are accounted for in our calculations.

[22] Because we are interested in all $>100 \mathrm{keV}$ precipitation, our results (Figures 4-8) are shown in terms of energy flux. Energy flux combines both the number and energy spectra of the precipitated electrons. A large number of $100 \mathrm{keV}$ electrons may produce a similar energy flux signature as a smaller number of $500 \mathrm{keV}$ electrons. However, the distribution of energetic electrons decreases rapidly with increasing electron energy. This effect partially explains why sources at geomagnetic latitudes $20^{\circ}$ include weak energy flux signatures despite high resonant electron energies (see below).

[23] Figure 4 displays $>100 \mathrm{keV}$ energy flux versus $L$ shell induced by a one-half second pulse for sources at geomagnetic latitudes of $15,25,35,45$, and 55 degrees that radiate $10,20,30$, and $40 \mathrm{kHz}$ waves. We first decouple frequency and geographic location by considering these transmitters to operate at a constant radiated power of 1 MW. We vary radiated power after this initial analysis. Keeping source latitude constant, Figure 4 shows that induced precipitation decreases with wave frequency above $L \simeq 1.7$, while the opposite is true for $L \simeq 1.7$. Furthermore, the region of illumination increases with decreasing wave frequency. In other words, $10 \mathrm{kHz}$ waves illuminate geomagnetic equatorial regions ranging from $L=1.5$ up to $L \simeq 3$, while $40 \mathrm{kHz}$ waves do not induce precipitation beyond $L \sim 2.3$ for any source location. At all locations, lower operating frequencies induce stronger precipitation peaks as well as total $>100 \mathrm{keV}$ energy flux.

[24] The various dependencies exhibited can be understood in terms of the resonance condition, disposition of raypaths through the magnetosphere, ionospheric absorption and Landau damping. According to equation (2), as wave frequency increases, the resonant parallel velocity decreases if everything else is held constant. Therefore $10 \mathrm{kHz}$ waves resonate with more energetic electrons than $40 \mathrm{kHz}$ waves, generally leading to stronger precipitation signatures for our chosen energetic electron distribution. For $L \lesssim 1.7$, however, $40 \mathrm{kHz}$ waves are more effective than $10 \mathrm{kHz}$ waves for two main reasons. First, higher wave frequencies deposit wave power flux at locations close to the Earth's surface. As seen in Figure 2, $40 \mathrm{kHz}$ rays bend sharply toward the Earth and do not propagate beyond $L \sim 2.3$. Second, at $L \lesssim 1.7$, the high gyrofrequency leads to $10 \mathrm{kHz}$ waves to be in resonance with very energetic electrons $(\gtrsim 500 \mathrm{keV})$, the trapped flux levels of which are relatively low. Above $L \simeq$ 1.7, the higher electron resonant energies combined with higher wave power flux at those locations yields stronger precipitation signatures for lower operating frequencies. In this same region, the stronger ionospheric attenuation and higher Landau damping with increasing frequency also contributes to cause $40 \mathrm{kHz}$ waves to generally induce weaker precipitation fluxes than $10 \mathrm{kHz}$ waves. For a given wave frequency, Figure 4 shows that there is an optimum source latitude from the point of view of precipitating $>100 \mathrm{keV}$ electrons. Sources located from $35^{\circ}$ to $45^{\circ}$ induce almost an order of magnitude higher $>100 \mathrm{keV}$ energy flux than those at $\leq 30^{\circ}$ or $\geq 50^{\circ}$.

[25] The location-dependence of the precipitation signatures can by clarified by studying Figure 1 and the resonance condition (equation (2)). As discussed above, rays injected from increasingly higher latitudes traverse the magnetosphere in a way such that $v_{z}$ along the raypath decreases. Therefore sources at, e.g., $55^{\circ}$, primarily resonate with $<100 \mathrm{keV}$ electrons (see Figure 1 and related discus- 

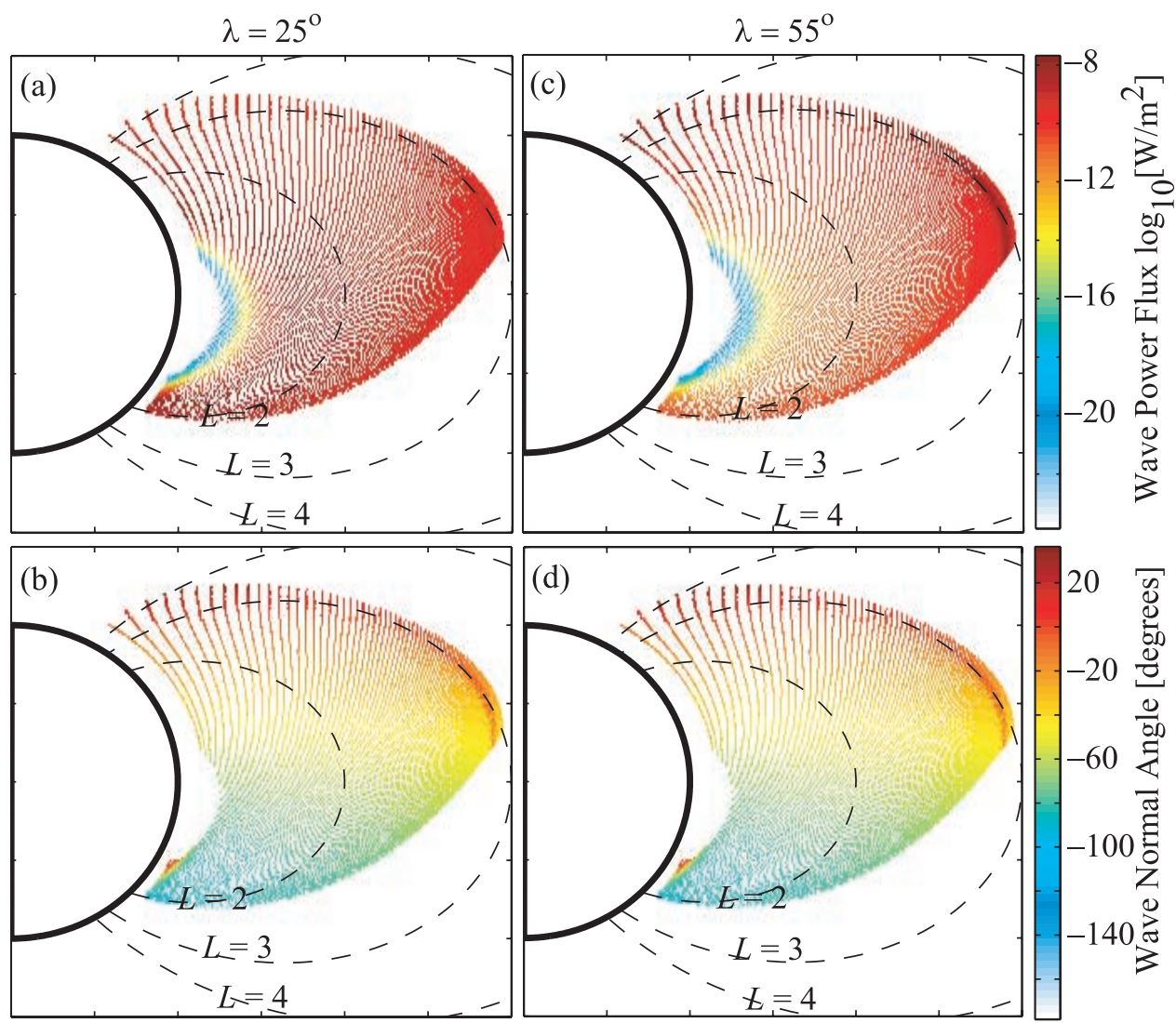

Figure 3. Wave power flux and wave normal angle distribution for $500 \mathrm{~kW}, 20 \mathrm{kHz}$ sources at geomagnetic latitudes of $25^{\circ}$ and $55^{\circ}$. These two wave properties, along with wave-particle resonance interactions, largely determine the precipitation signatures shown here.

sion). The relatively weak precipitation induced by sources at $15^{\circ}$ and $25^{\circ}$ is likely due to three factors. First, there are relatively fewer electrons at the very high resonant energies found at low $L$-shells. Second, the powerful rays injected close to the source site have very short raypaths before reaching the southern hemisphere. Finally, ionospheric absorption given by Helliwell [1965, Figures 3-35] increases at low latitudes. These reasons, which result in weak $>100 \mathrm{keV}$ precipitation signatures from sources at low geomagnetic latitudes, plus the small $v_{z}$ for sources at high

证

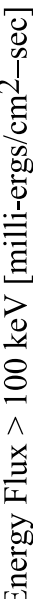
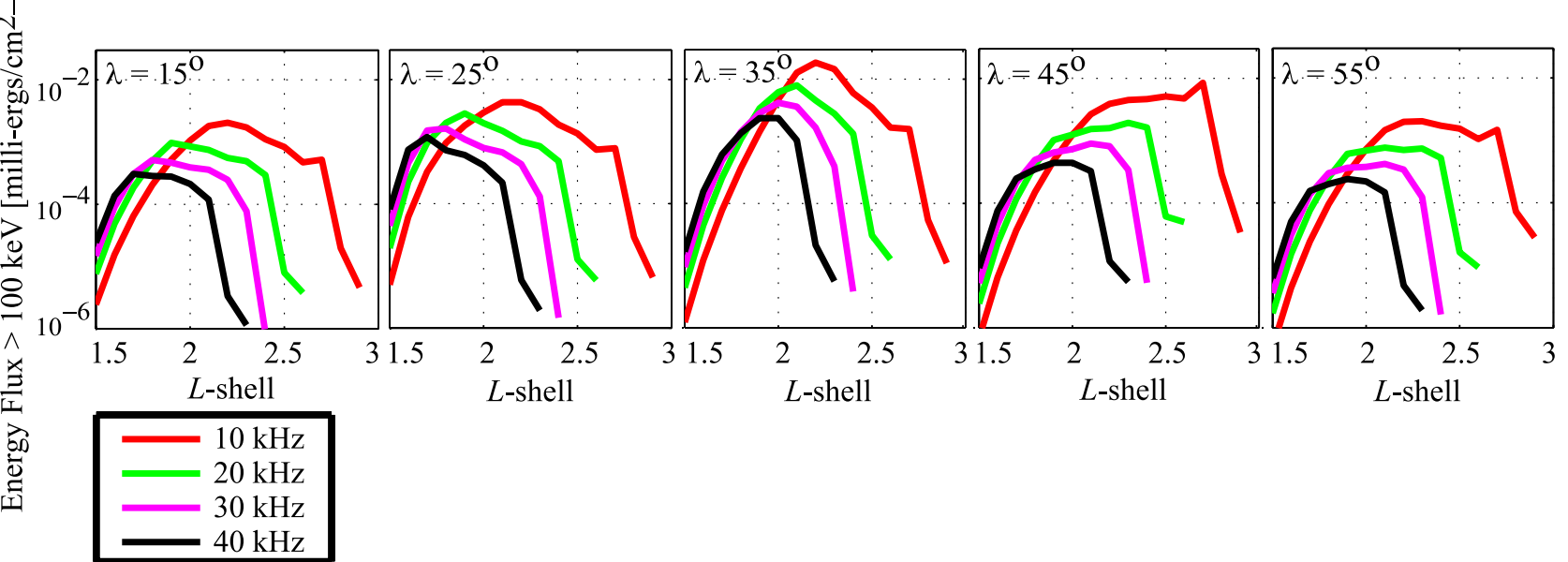

Figure 4. Energy flux $>100 \mathrm{keV}$ versus $L$-shell for hypothetical transmitters located at $\lambda=15^{\circ}, 25^{\circ}$, $35^{\circ}, 45^{\circ}$, and $55^{\circ}$, for operating frequencies of $10,20,30$, and $40 \mathrm{kHz}$. Each transmitter radiates at $1 \mathrm{MW}$. Note that sources located at $\lambda=35^{\circ}-45^{\circ}$ induce almost an order of magnitude more energetic electron precipitation than other locations. In all cases induced precipitated flux increases as the operating frequency decreases. 


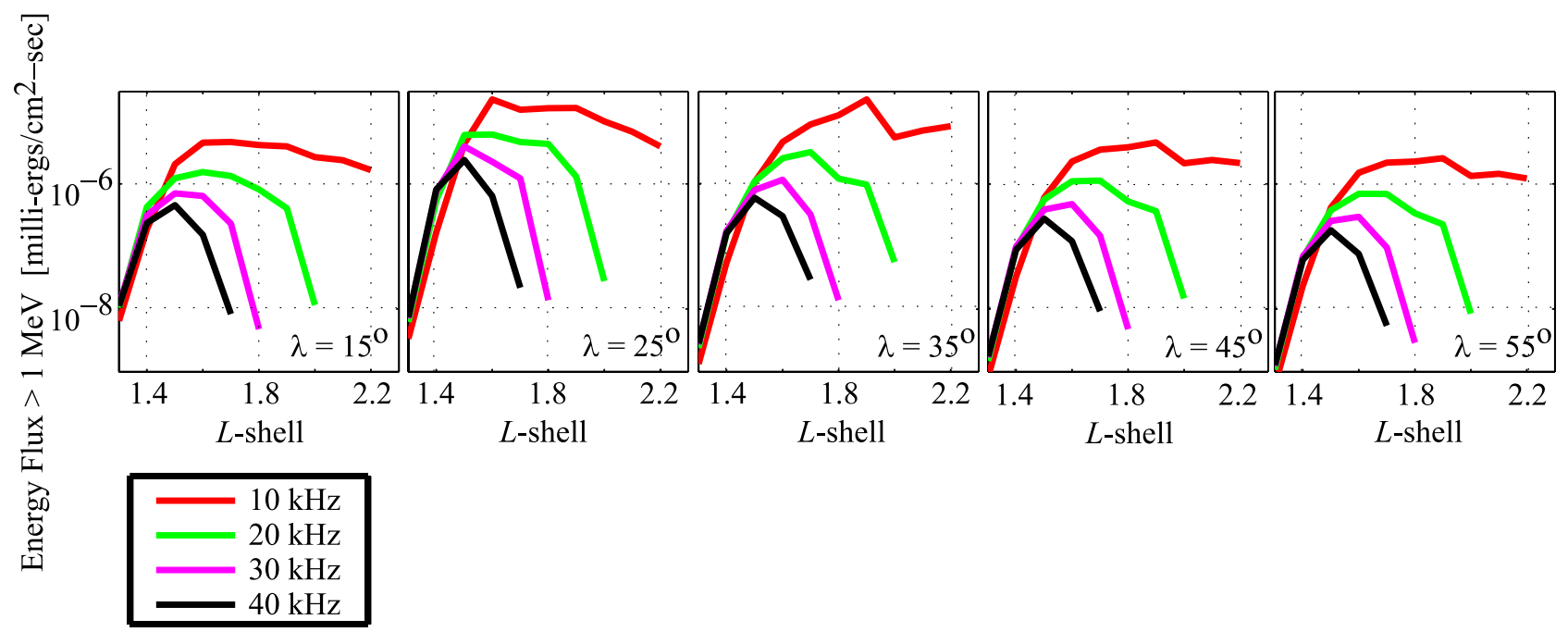

Figure 5. Energy flux $>1 \mathrm{MeV}$ versus $L$-shell for hypothetical transmitters located at $\lambda=15^{\circ}, 25^{\circ}, 35^{\circ}$, $45^{\circ}$, and $55^{\circ}$, for operating frequencies of $10,20,30$, and $40 \mathrm{kHz}$. Each transmitter radiates at $1 \mathrm{MW}$. Note that sources located at $\lambda=25^{\circ}-35^{\circ}$ induces the most $>1 \mathrm{MeV}$ precipitation, locations lower in geomagnetic latitude than for $>100 \mathrm{keV}$ precipitation.

latitudes, explain why midlatitude source locations maximally precipitate $>100 \mathrm{keV}$ electrons.

[26] The discussion above highlights that source location, more than operating frequency, impacts $>100 \mathrm{keV}$ electron precipitation. In fact, our results show that relatively ineffective $40 \mathrm{kHz}$ waves injected from a source at $35^{\circ}$ induces a stronger $>100 \mathrm{keV}$ precipitation peak than $20 \mathrm{kHz}$ waves from a source at $15^{\circ}, 25^{\circ}$ or $55^{\circ}$.

[27] If we consider instead $>1 \mathrm{MeV}$ electron precipitation, Figure 5 shows a similar trend, with source location once again being more important than operating frequency. In this case, however, the optimum location is from $25^{\circ}$ to $35^{\circ}$ latitude, as opposed to $35^{\circ}$ to $45^{\circ}$ for $>100 \mathrm{keV}$ electrons, because resonant energy near the magnetic equator is higher on $L$-shells closer to the Earth. Thus the strongest $>1 \mathrm{MeV}$ electron precipitation signature is produced by transmitters located at lower latitudes than sources than those that induce the strongest $>100 \mathrm{keV}$ energy flux. Also, the absolute energy flux levels are three to four orders of magnitude lower than in Figure 5 because the typical population of trapped electrons contain much fewer $>1 \mathrm{MeV}$ than $>100 \mathrm{keV}$ electrons. Finally, we note that both Figures 4 and 5 display identical trends for induced energy flux versus operating frequency.

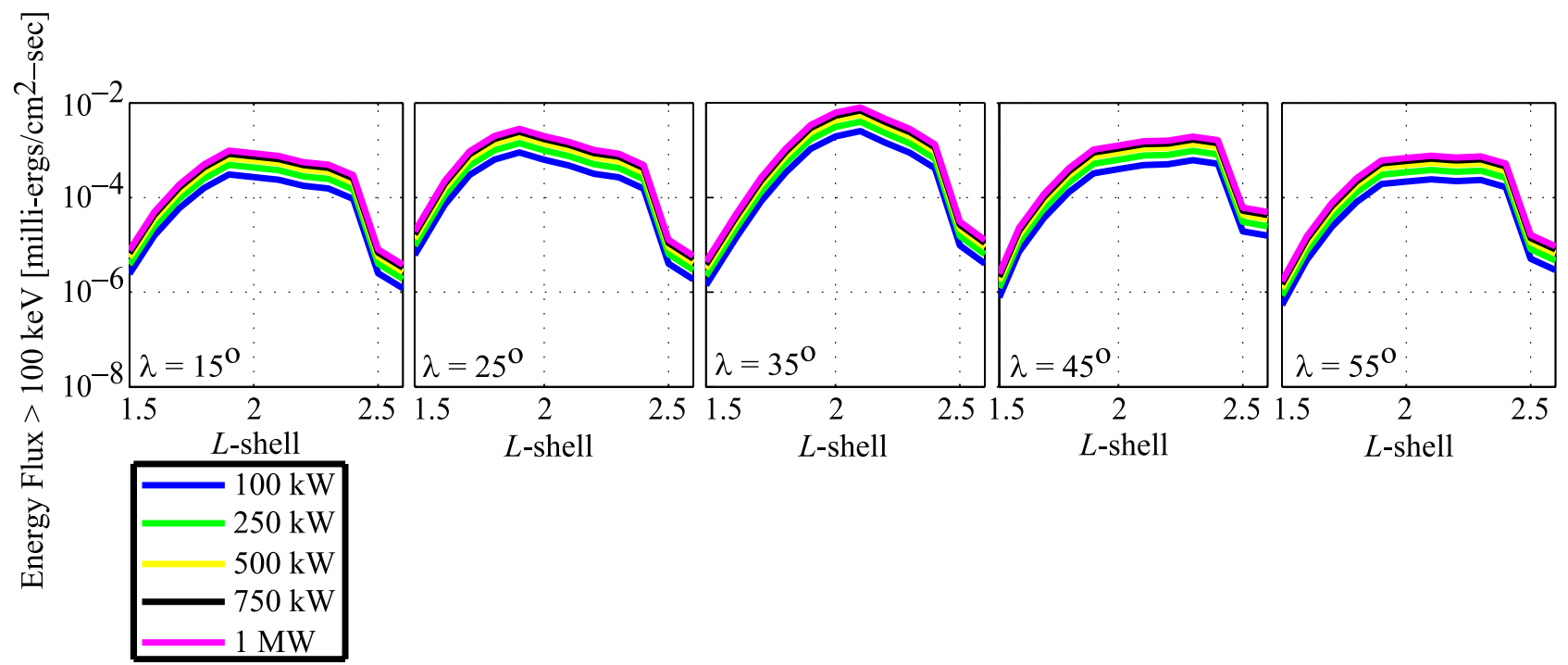

Figure 6. Energy flux $>100 \mathrm{keV}$ versus $L$-shell for hypothetical sources distributed in geomagnetic latitude as in Figures 4 and 5. We select a single operating frequency of $20 \mathrm{kHz}$, and radiated power levels of $100 \mathrm{~kW}, 250 \mathrm{~kW}, 500 \mathrm{~kW}, 750 \mathrm{~kW}$, and $1 \mathrm{MW}$. Observe that while high radiated power can compensate for poor location, source location generally exerts more influences on the precipitation signature. 

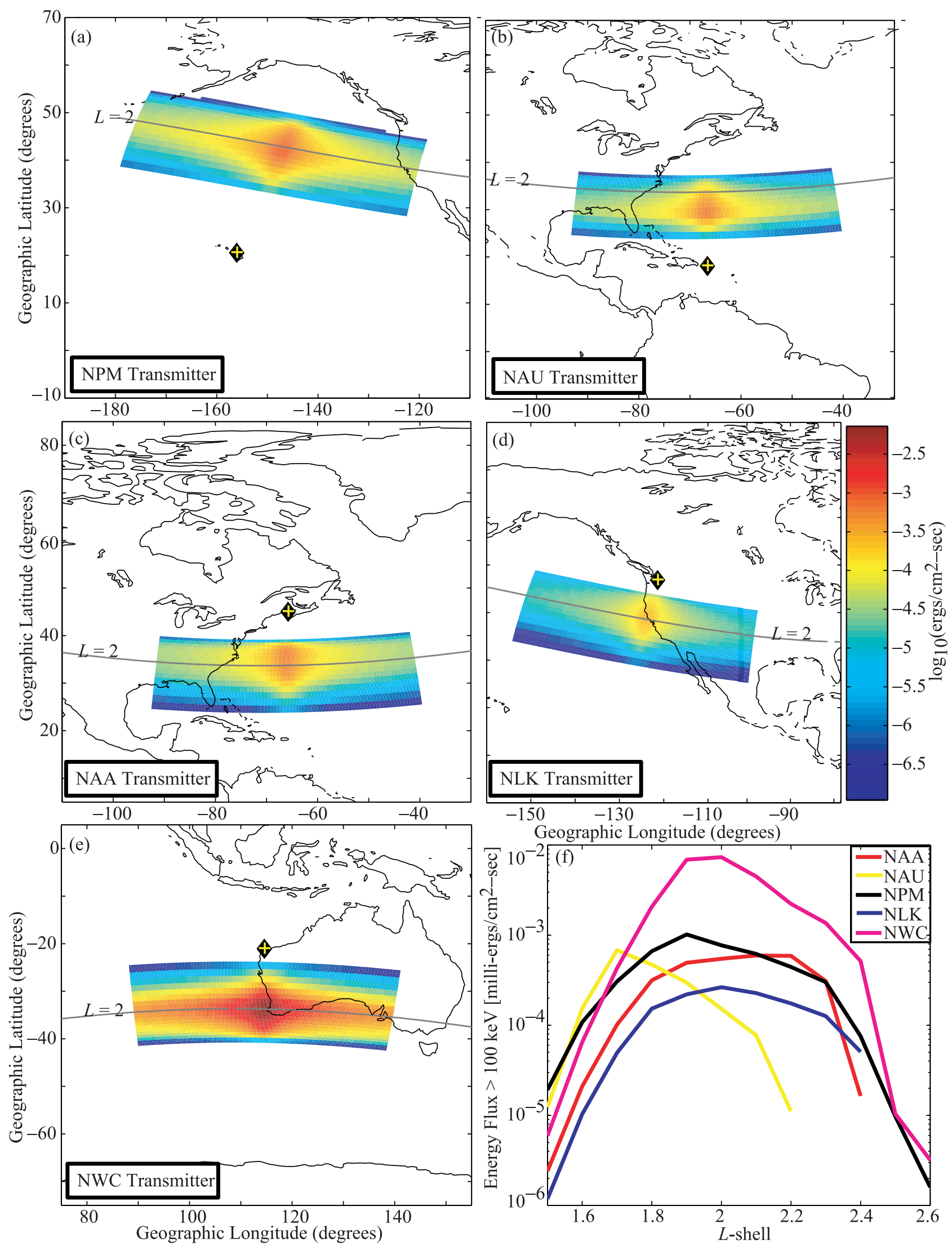

Figure 7. (a)-(e) Energetic electron precipitation zones on Earth for the five existing VLF transmitters considered in this study. A common color bar is shown on the right middle panel next to the NLK transmitter. (f) Energy flux $>100 \mathrm{keV}$ for the five existing VLF transmitters. NWC induces the strongest precipitation signature. 


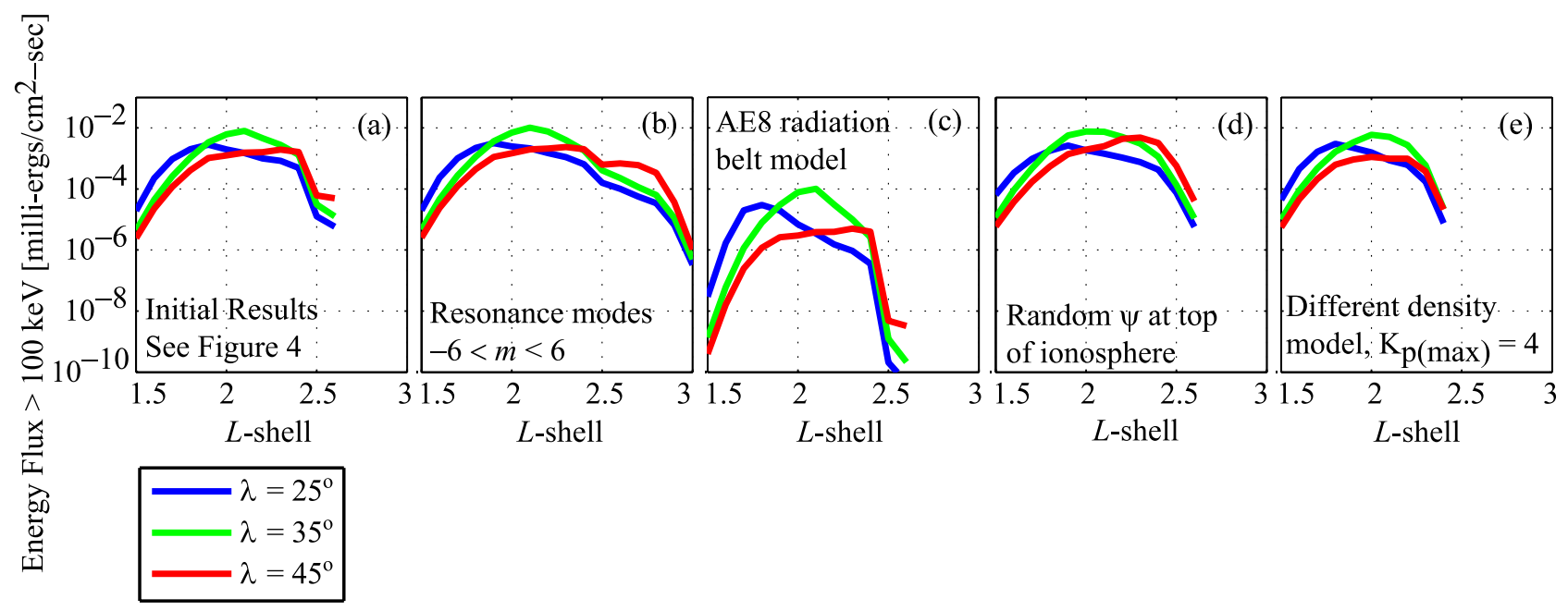

Figure 8. (a) A plot reproduced from Figure $4 \mathrm{a} 20 \mathrm{kHz}, 1 \mathrm{MW}$ source at $25^{\circ}, 35^{\circ}$, and $45^{\circ}$. (b) Similar to Figure $8 \mathrm{a}$, but including resonance modes $-5 \leq m \leq 5$. There is significantly greater precipitation at $L \gtrsim 2.6$. (c) The AE8 radiation belt model, with an assumed sinusoidal loss cone was used to calculate the precipitation signature here. Our results are very sensitive to assumed pitch angle loss cone distribution. (d) Precipitation signature accounting for randomized initial wave normal angle. The results are almost identical to those in Figure 8a. (e) Results for a plasmapause located at $L=3.8$. Precipitation stops at $L \sim 2.4$, as opposed to $L \sim 2.6$ in Figure 8a.

[28] Having investigated the importance of source location versus operating frequency at precipitating energetic electrons, we now turn to radiated power. We select a single frequency of $20 \mathrm{kHz}$, the same source latitudes used above, and radiated power levels of $100 \mathrm{~kW}, 250 \mathrm{~kW}, 500 \mathrm{~kW}$, $750 \mathrm{~kW}$, and $1 \mathrm{MW}$. Again, we consider only the $m=1$ counterstreaming resonance, include latitude-dependent ionospheric absorption, and calculate pitch angle scattering for resonant, loss cone electrons. Figure 6 shows the results of these calculations. We first observe that sources located locations of $35^{\circ}-45^{\circ}$ once again induce the strongest $>100 \mathrm{keV}$ energy flux for a given radiated power. Changing the power level from $100 \mathrm{~kW}$ to $1 \mathrm{MW}$ has a much smaller effect on the precipitation signature than changing the frequency from $10 \mathrm{kHz}$ to $40 \mathrm{kHz}$ (see Figure 4). Figure 6 again highlights the importance of source location over other parameters, although high radiated power levels can sometimes compensate. The second and third panels show that a $1 \mathrm{MW}$ source at $25^{\circ}$ precipitates approximately as many electrons as a $100 \mathrm{~kW}$ source at $35^{\circ}$. A $100 \mathrm{~kW}$ source at $35^{\circ}$, however, precipitates more $>100 \mathrm{keV}$ electrons than a $1 \mathrm{MW}$ source at either $15^{\circ}$ or $55^{\circ}$.

\subsection{Existing VLF Transmitters}

[29] Based on the above analysis, we can now quantify the effectiveness of the existing ground-based VLF sources at precipitating $>100 \mathrm{keV}$ electrons. Inspecting Table 1, we predict that NWC induces the strongest precipitation signature because of its excellent location $\left(31.7^{\circ}\right)$, the lowest operating frequency of all sources $(19.8 \mathrm{kHz})$ and high radiated power of $1 \mathrm{MW}$. NLK, on the other hand, should induce the least energetic electron precipitation because it is located at a high latitude and operates with relatively low radiated power. Although it is somewhat difficult to specifically rank the remaining three sources, we can still make some observations. NAU does not induce precipitation beyond $L \simeq 2.3$ because it operates at $40.75 \mathrm{kHz}$, the raypaths for which do not reach the equatorial plane for $L>$ 2.3. NAU is at an excellent location $\left(28.6^{\circ}\right)$ for precipitating electrons, but radiates at only $100 \mathrm{~kW}$. NAA has a more optimum frequency $(24 \mathrm{kHz})$ and high radiated power, but is at a less optimum location, 54.6 . NPM is located at $21.4^{\circ}$, which is a better location than NAA. NPM also operates at a lower frequency and a relatively high radiated power of $424 \mathrm{~kW}$. We predict that all three sources induce approximately equal levels of energetic electron precipitation.

[30] In addition to energy flux versus $L$-shell shown above, we also present plots of the induced precipitation zones on the Earth's surface, with the signatures extrapolated in longitude as described above and by Bortnik et al. [2006b]. Figure 7 displays these results for the NAA, NLK, NAU, NPM, and NWC ground-based VLF transmitters, using the characteristics listed in Table 1. Our predictions made above were essentially correct, although NPM does end up precipitating more $>100 \mathrm{keV}$ electrons than NAU. The NWC transmitter located in Western Australia induces by far the strongest precipitation signature, due primarily to its optimum location $\left(31.7^{\circ}\right)$, low operating frequency and high radiated power. The NPM transmitter induces the second strongest precipitation signature because of its location, relatively low operating frequency of $21.4 \mathrm{kHz}$, and high radiated power. NAU has a slightly stronger precipitation peak than NAA because of its more favorable location, even though NAA radiates at higher power levels and at a lower frequency $(24 \mathrm{kHz}$ versus $40.75 \mathrm{kHz})$. NAU, however, illuminates a very narrow region of the magnetosphere (only up to $L \sim 2.2$ ), while NAA precipitates electrons up to $L \sim 2.4$. The NAU and NAA sources again highlight the dominant role of source location in precipitating $>100 \mathrm{keV}$ electrons. The NLK transmitter has very similar characteristics to NAA but operates at a lower 
radiated power and therefore induces the least energetic electron precipitation of all the transmitters considered.

[31] Note, once again, that the plots in Figure 7 display spatial regions of $>100 \mathrm{keV}$ induced electron precipitation. If we consider all energies, the precipitation zones would extend farther in $L$-shell because resonant energy is lower at the lower equatorial electron gyrofrequencies farther away from the Earth's surface. Finally, we should observe that the transmitters do not induce appreciable precipitation of $>100 \mathrm{keV}$ electrons beyond $L \sim 2.6$, consistent with the analysis from the comprehensive study by Abel and Thorne [1998a], which concluded that ground-based VLF sources have a significant effect on electron lifetimes at $L \lesssim 2.6$.

\subsection{Sensitivity to Assumptions}

[32] The results presented thus far are based on a number of assumptions that, if changed, may modify our conclusions. Specifically, we have neglected higher order resonance modes, assumed a square-loss cone edge instead of a sinusoidal one, ignored ionospheric density irregularities that would randomize the initial wave normal angle, and considered a quiet time magnetosphere with a plasmapause at $L=5.5$. In Figure 8, we examine each of these assumptions in turn. We select a single operating frequency of $20 \mathrm{kHz}$ and calculate $>100 \mathrm{keV}$ electron precipitation for sources located at $25^{\circ}, 35^{\circ}$, and $45^{\circ}$. All sources were operated at $1 \mathrm{MW}$ of power, and except for Figure 8b, we considered only the $m=1$ counterstreaming resonance. For reference, panel (a) shows the results initially shown in Figure 4.

[33] Figure $8 \mathrm{~b}$ shows the variation of induced precipitation if several higher order resonances, from $-5 \leq m \leq 5$, are included. As the absolute value of the resonance mode increases, the electron resonance energy also increases. Furthermore, higher-order wave-particle interactions are less effective than the fundamental $m=1$ mode. As can be seen in Figure 8b, the $L$-shell range of the precipitation region increases up to $L \sim 3$, as opposed to $L \sim 2.6$ before including additional modes. At higher $L$-shells, the low gyrofrequency leads to resonance with $<100 \mathrm{keV}$ electrons if we only include $m=1$ (see Figure 1). Our results therefore do not accurately determine the absolute values of precipitation fluxes at these $L$-shells. However, we should highlight that total precipitation from $1.5 \lesssim L \lesssim 2.6$ is approximately the same in both cases and our approach appears to be valid in that region.

[34] The third panel shows the effect of adopting the AE8 radiation belt model [Vette, 1991] with an assumed sinusoidal pitch angle distribution. For a given electron energy level $>100 \mathrm{keV}$, the AE8 electron flux is about 1 to 2 orders of magnitude higher than the model we used earlier. Nevertheless, Figure 8c shows the AE8 radiation belt model results in a substantially weaker (2 to 4 orders of magnitude) precipitation signature at a similar $L$-shell range as before. This result highlights that induced electron precipitation is very sensitive to the assumed pitch angle distribution. The precipitation signatures shown above are therefore more appropriate for disturbed geomagnetic conditions when the pitch angle distribution may be filled with electrons at all pitch angles up to the loss cone. In this connection, however, we note that the only parameter affected by the shape of the near-loss cone pitch angle distribution is the absolute precipitation flux levels. All other parameter dependencies and comparisons (e.g., between transmitters at different locations) as discussed above are still valid, and relatively independent of the near-loss cone pitch angle distribution.

[35] The final two panels show the effect of including ionospheric density irregularities that results in randomized initial wave normal angles, and more active geomagnetic conditions with $K_{p(\max )}=4$ and a plasmapause at $L=3.8$ (instead of $K_{p(\max )}=0$ and a plasmapause at $\left.L=5.5\right)$. For the former case, each of the rays injected at the top of the ionosphere was assigned a random number for its initial wave normal angle, uniformly distributed between $-30^{\circ}$ and $30^{\circ}$ about the local vertical direction. In both scenarios, the precipitation signatures are similar to that in panel (a), although a closer plasmapause results in precipitation only up to $L \sim 2.4$ (instead of $L \sim 2.6$ in (a)). Although individual raypaths and $k$-vectors do change with these parameters, the differences are drowned out by the effect of injecting several hundred rays from $10^{\circ}$ to $60^{\circ}$. The total spectrum of raypaths and $k$-vector distribution is approximately the same in the end because the rays fill the inner magnetosphere with wave power up to $L \sim 2.5$ regardless of, e.g., initial wave normal angle of the individual rays.

\section{Summary and Conclusions}

[36] We have calculated the $>100 \mathrm{keV}$ energetic electron precipitation that would be induced by several hypothetical ground-based VLF sources distributed broadly in geomagnetic latitude and at a wide range of operating frequencies. The sources radiation patterns were approximated as that of a short electric dipole, and we used the formulation described by Rodriguez [1994, p. 65] to determine attenuation from each source at $100 \mathrm{~km}$ from $10^{\circ}$ to $60^{\circ}$ in geomagnetic latitude. The wave power at this point was then attenuated through the ionosphere with a latitude and frequencydependent absorption factor in accordance with Figures 335 by Helliwell [1965]. We then used the Stanford VLF raytracing program [Inan and Bell, 1977] to determine raypaths through the inner magnetosphere, and also calculated path-integrated Landau damping. Finally, we used the methodology described by Bortnik et al. [2006a] to determine the precipitated flux of energetic electrons, based on a square (sharp) near loss cone pitch angle distribution for the first order $m=1$ counterstreaming resonance.

[37] We investigated the dependence of the precipitation on three major parameters: source location, operating frequency and radiated power. Specifically, we considered sources at geomagnetic latitudes of $15^{\circ}, 25^{\circ}, 35^{\circ}, 45^{\circ}$, and $55^{\circ}$, operating frequencies of $10 \mathrm{kHz}, 20 \mathrm{kHz}, 30 \mathrm{kHz}$, and $40 \mathrm{kHz}$, and radiated powers of $100 \mathrm{~kW}, 250 \mathrm{~kW}$, $500 \mathrm{~kW}, 750 \mathrm{~kW}$, and $1 \mathrm{MW}$. Source location, more than any other factor, affects $>100 \mathrm{keV}$ electron precipitation. Sources located at $35^{\circ}$ to $45^{\circ}$ induce almost an order of magnitude more $>100 \mathrm{keV}$ electron precipitation than sources located at $15^{\circ}, 25^{\circ}$, or $55^{\circ}$. For a given location, operating frequencies of 10 to $20 \mathrm{kHz}$ are more effective than 30 to $40 \mathrm{kHz}$, with $10 \mathrm{kHz}$ waves producing the strongest precipitation signatures. An operating frequency of $10 \mathrm{kHz}$ also extends the precipitation zone up to $L 3$. For $>1 \mathrm{MeV}$ electrons, transmitters that radiate $10 \mathrm{kHz}$ waves at 
$25^{\circ}$ to $35^{\circ}$ latitude induce the strongest precipitation signature. All of our simulation results included the effects of stronger ionospheric absorption at lower latitudes and higher frequencies, and we accounted for the different wave power flux and $k$-vector distributions that resulted from the various configurations.

[38] We also showed results for five existing groundbased VLF sources: the NAA, NLK, NAU, NPM, and NWC transmitters (see Table 1 for information). We can rank the sources, from most to least effective, at inducing $>100 \mathrm{keV}$ electron precipitation: NWC, NPM, NAU, NAA, and NLK. Our analysis based on hypothetical VLF transmitters allowed us to explain these results in terms of the three major parameters analyzed. NWC induces the strongest signature due to its optimal source location, low operating frequency, and high radiated power. NLK, on the other hand, is at a very high geomagnetic latitude, and thus rays injected from that source tend to resonate mostly with $<100 \mathrm{keV}$ electrons. Moreover, NLK radiates at a relatively low power level of $192 \mathrm{~kW}$. The precipitation signature strength of the remaining transmitters fall in between those two and can be explained with a similar analysis. It is also interesting to note that our results do not show precipitation beyond $L \sim 2.6$, which agrees with the conclusions from the landmark study of Abel and Thorne [1998a] that ground-based VLF sources impact electron lifetimes below $L \sim 2.6$.

[39] We also investigated the sensitivity of our results to our model assumptions. We considered ionospheric density irregularities that may change the initial wave normal angle at injection, and different magnetospheric conditions that would alter the raypaths and $k$-vector distribution. The precipitation signatures are relatively insensitive to these changes, with total flux and $L$-shell distribution showing no significant difference. If we include higher-order resonance modes, the precipitation region extends significantly, up to $L \sim 3$. Up to $L \sim 2.6$, however, including only the $m=1$ mode appears to properly calculate the induced precipitation. Adopting the AE8 radiation belt model, with an assumed sinusoidal loss cone, lowers the $>100 \mathrm{keV}$ electron precipitation by 2 to 4 orders of magnitude.

[40] Future research in this topic should examine the effect of magnetic field aligned density enhancements, further investigate different radiation belt models, quantify the effects of rays formed by the shadow boundary in the conjugate hemisphere, and determine how refractive effects alter the propagation and power flux characteristics shown here. Such differences could modify the fine details of global signatures of transmitter induced electron precipitation shown here.

[41] Acknowledgments. This research was supported by DARPA, the Air Force Research Laboratory (AFRL), and the HF Active Auroral Research Program (HAARP) under contract F19628-03-C-0059-P000003 and by the Office of Naval Research (ONR) under grant N00014-07-10789.
[42] Zuyin Pu thanks Michael J. Starks and another reviewer for their assistance in evaluating this paper.

\section{References}

Abel, B., and R. M. Thorne (1998a), Electron scattering loss in the Earth's inner magnetosphere: 1. Dominant physical processes, J. Geophys. Res., 103, 2385. (Correction, J. Geophys. Res., 104, 4627, 1999)

Abel, B., and R. M. Thorne (1998b), Electron scattering loss in the Earth's inner magnetosphere: 2 . Sensitivity to model parameters, J. Geophys. Res., 103, 2397, (Correction, J. Geophys. Res., 104, 4627, 1999)

Angerami, J. J., and J. O. Thomas (1964), Studies of planetary Atmospheres: 1. The distribution of electrons and ions in the Earth's exosphere, J. Geophys. Res., 69(21), 4537-4560.

Bell, T. F. (1984), The nonlinear gyroresonance interaction between energetic electrons and coherent VLF waves propagating at an arbitrary angle with respect to the Earth's magnetic field, J. Geophys. Res., 89(A2), 905-918.

Bell, T. F., U. S. Inan, J. Bortnik, and J. D. Scudder (2002), The Landau damping of magnetospherically reflected whistlers within the plasmasphere, Geophys. Res. Lett., 29(15), 1733, doi:10.1029/2002GL014752.

Bortnik, J., U. S. Inan, and T. F. Bell (2006a), Temporal signatures of radiation belt electron precipitation induced by lightning generated MR whistler waves: 1. Methodology, J. Geophys. Res., 111, A02204, doi:10.1029/2005JA011182

Bortnik, J., U. S. Inan, and T. F. Bell (2006b), Temporal signatures of radiation belt electron precipitation induced by lightning generated MR whistler waves: 2. Global signatures, J. Geophys. Res., 111, A02205, doi:10.1029/2005JA011398.

Carpenter, D. L., and R. R. Anderson (1992), An ISEE/Whistler model of equatorial electron-density in the magnetosphere, J. Geophys. Res., 97(A2), 1097-1108.

Helliwell, R. A. (1965), Whistlers and Related Ionospheric Phenomena, Stanford Univ. Press, Stanford, Calif.

Inan, U. S., and T. F. Bell (1977), The plasmapause as a VLF waveguide, J. Geophys. Res., 82, 2819.

Inan, U. S., and T. F. Bell (1991), Pitch angle scattering of energetic particles by oblique whistler waves, Geophys. Res. Lett., 19(18), 49-52.

Inan, U. S., and S. Tkalcevic (1982), Nonlinear equations of motion for Landau response interactions with a whistler mode wave, J. Geophys. Res., 87(A4), 2363-2367.

Inan, U. S., H. C. Chang, and R. A. Helliwell (1984), Electron-precipitation zones around major ground-based VLF signal sources, J. Geophys. Res., 89(A5), 2891-2906.

Kennel, C. F., and H. E. Petschek (1966), Limit on stably trapped particle fluxes, J. Geophys. Res., 71(1), 1-28.

Lyons, L. R., R. M. Thorne, and C. F. Kennel (1972), Pitch angle diffusion of radiation belt electrons within the plasmasphere, J. Geophys. Res., 77(19), 3455-3474.

Rodriguez, J. V. (1994), Modification of the Earth's ionosphere by very-lowfrequency transmitters, Stanford University thesis, Stanford, Calif.

Rodriguez, J. V., U. S. Inan, and T. F. Bell (1994), Heating of the nighttime $D$ region by very low frequency transmitters, J. Geophys. Res., 99(A12), 23,329-23,338

Shprits, Y. Y., W. Li, and R. M. Thorne (2006), Controlling effect of the pitch angle scattering rates near the edge of the loss cone on electron lifetimes, J. Geophys. Res., 111, A12206, doi:10.1029/2006JA011758.

Vette, J. (1991), The AE-8 trapped electron model environment, Report 91-24, Natl. Space Sci. Data Cent., Greenbelt, Md.

Walt, M. (1994), Introduction to Geomagnetically Trapped Radiation, Cambridge Univ. Press, New York.

T. F. Bell, U. S. Inan, and P. Kulkarni, STAR Laboratory, Stanford University, 308 Packard Building, MC 9515, 350 Serra Mall, Stanford, CA 94305, USA. (pxk161@stanford.edu)

J. Bortnik, Department of Atmospheric and Oceanic Sciences, University of California, Room 7115, Math Sciences Bldg., UC Los Angeles, CA 90095-1565, USA. 